\title{
SIMULACRO ODONTOPEDIÁTRICO COM RECURSO À ESTRATÉGIA DE ENSINO ROLEPLAYING EM EDUCAÇÃO PARA A SAÚDE ORAL
}

\author{
Maria do Rosário Dias ${ }^{1}$ \\ https://orcid.org/0000-0003-3888-9688 \\ Ana Antunes ${ }^{2}$ \\ https://orcid.org/0000-0001-8128-3142 \\ Ana Dias ${ }^{3}$ \\ https://orcid.org/0000-0001-7420-3925 \\ Beatriz Duarte ${ }^{4}$ \\ https://orcid.org/0000-0001-5334-442X \\ Maria Calejo Pires ${ }^{5}$ \\ https://orcid.org/0000-0002-3047-5088
}

\section{RESUMO}

O presente artigo tem como objetivo desmistificar no âmbito de um ato pedagógico, i) o acontecimento desenvolvimentista da erupção dos dentes definitivos na criança; ii) sedimentar precocemente hábitos salutogénicos; e iii) elaborar instrumentos de Educação para a Saúde, evidenciando-se, assim, a importância da comunicação relacional e terapêutica no setting terapêutico de Medicina Dentária. As bases teóricas foram fundamentadas de acordo com Chernoff (1973); Dranka (2012); Magalhães et al. (2013); Mota-Cardoso (2012); Santos (2017). Foi realizado pelos discentes de um simulacro terapêutico-pedagógico em setting odontopediátrico com recurso à técnica do roleplaying, consolidando a vivência de um vínculo terapêutico, a acessibilidade e o fortalecimento dos discentes, no contexto da aprendizagem das suas capacidades comunicacionais. É consentâneo que o roleplaying, enquanto estratégia de ensinoaprendizagem permite que os discentes edifiquem conhecimentos sobre as suas (auto) capacidades comunicacionais a partir de uma reflexão crítica das suas próprias experiências e/ou dos seus pares.

Palavras-Chave: Dentição Decídua; Dentição Definitiva; Educação para a Saúde Oral; Pictogramas; Roleplaying

Simulacro odontopediátrico con recurso a la estrategia de enseñanza del juego de roles en la educación para la salud oral.

\section{RESUMEN}

El presente artículo tiene como objetivo desmitificar en el ámbito de un acto pedagógico, i) el acontecimiento de desarrollo de la erupción de los dientes definitivos en el niño; ii) consolidar hábitos salutogénicos precozmente; y iii) elaborar instrumentos de Educación para la Salud, evidenciándose, así, la importancia de la comunicación relacional y terapéutica en el entorno terapéutico de la Medicina odontológica. Las bases teóricas fueron fundamentadas de acuerdo con Chernoff (1973); Dranka (2012); Magalhães et al. (2013); Mota-Cardoso (2012); Santos (2017). Fue realizado por los estudiantes un simulacro terapéutico-pedagógico en el entorno odontopediátrico con recurso a la técnica del juego de roles, consolidando la experiencia de un vínculo terapéutico, la admisión, el empoderamiento de los estudiantes, en el contexto de aprendizaje de sus capacidades de comunicación. Es coherente que el juego de roles, como estrategia de enseñanza-aprendizaje permite a los estudiantes desarrollar conocimientos sobre sus (auto) capacidades de comunicación a partir de una reflexión crítica de sus propias experiencias y/o de sus compañeros.

Palabras Clave: Dentición temporal; Dentición Definitiva; Educación para la Salud Oral; Pictogramas; Juego de rol.

Pediatric Dentistry Simulacrum using the roleplaying strategy in Oral Health Education.

\section{ABSTRACT}

The aim of this article is to demystify within the scope of a pedagogical act, i) the eruption of permanent teeth in the child; ii) establish salutogenic habits from an early age; and iii) create communication and health education tools, thus

\footnotetext{
${ }^{1}$ Egaz Moniz, Cooperativa de Ensino Superior, Portugal. Email: mariadorosario.dias@gmail.com

${ }^{2}$ Estudante Egaz Moniz, Cooperativa de Ensino Superior, Portugal. Email: Abaantunes7@hotmail.com

${ }^{3}$ Estudante Egaz Moniz, Cooperativa de Ensino Superior, Portugal. Email: Ritafonseca.1999@gmail.com

${ }^{4}$ Estudante Egaz Moniz, Cooperativa de Ensino Superior, Portugal. Email: Beatriz.duarte.eslc@gmail.com

${ }^{5}$ Investigadora Auxiliar Egaz Moniz, Cooperativa de Ensino Superior, Portugal. Email: calejopires.maria98@gmail.com
} 
evidencing the importance of both relational and therapeutic communication of Dental Medicine. The theoretical bases were founded according to Chernoff, (1973), Dranka (2012), Magalhães et al. (2013), Mota-Cardoso (2012) and Santos (2017). A therapeutic-pedagogical simulation in the pediatric dental environment was carried out by the students with recourse to the role-playing technique, consolidating the experience of a therapeutic bond, the admission, the empowerment of the students, in the context of learning their communication. It is coherent that the role play, as a teaching-learning strategy, allows students to develop knowledge about their (self) communication skills from a critical reflection of their own experiences and / or of their classmates.

Keywords: Deciduous Dentition; Permanent Dentition; Oral Health Education; Pictograms; Roleplaying.

\section{Introdução}

A comunicação interpessoal é uma necessidade humana básica, o denominador comum de todas as relações estabelecidas pelos Profissionais de Saúde, independente da sua formação profissional, constituindo-se como um instrumento chave para a qualidade do procedimento terapêutico (Albuquerque, 2013; Araújo et al., 2007; Mota-Cardoso, 2012; Dougall \& Fiske, 2008).

Relativamente à natureza da comunicação humana podemos dividi-la em duas componentes essenciais- verbal e não-verbal- sendo que, não são mutuamente exclusivas, emergindo, na generalidade, de forma combinada e interligada, verificando-se, também que, a maioria dos autores, assumem que 90\% da comunicação face a face é não-verbal (Dias et al., 2014; Mota-Cardoso, 2012; Ramos \& Bortagarai, 2012).

O presente estudo de caso, decorre na trajetória de um ato terapêutico simulado que está dividido em dois momentos distintos- ato clínico e ato pedagógico- tendo como objetivo a promoção, prevenção e manutenção da saúde oral do Paciente, suportado na análise da comunicação, verbal e não-verbal, da díade Médico-Dentista-Paciente.

As crianças são quase inteiramente dependentes dos adultos para se deslocarem às consultas de Medicina Dentária e, a sua presença, na qualidade de pacientes de $2^{\mathrm{a}}$ ordem é fundamental para fornecer a informação adequada para a concretização das intervenções clínicas (Silva, 2018). No entanto, conhecer e admitir a especificidade da Criança a nível emocional, cognitivo e social e, adaptar a essência da comunicação relacional é essencial para o desenvolvimento de uma aliança terapêutica vinculativa (Armelin et al., 2005; Mota-Cardoso, 2012).

De acordo com a American Academy of Pediatric Dentistry (AAPD, 2014) e a American Dental Association (ADA, 2000), é recomendado que a primeira visita ao Médico-Dentista seja efectuada entre os 6 meses no decurso da primeira erupção dentária e, nunca após os 12 meses de idade, possibilitando, assim, o diagnóstico precoce de lesões e a avaliação do desenvolvimento crânio-facial da criança. Contudo, varios estudos são unanimes em afirmar que, a maioria das crianças realiza, tardiamente, a sua primeira ida ao Médico-Dentista e, na maioria dos casos, apenas por necessidades de urgência de terapêutica sintomatológica (Comassetto et al., 2019; Dias et al., 2019a; Graça, 2012; Lemos et al., 2014; Mika et al., 2018).

A experiência Odontopediátrica é sustentada, a priori, numa comunicação relacional e no intercâmbio de informações pedagógicas permutadas entre a Criança e o Médico Dentista. Uma comunicação relacional adequada, permite, assim, favorecer o vínculo terapêutico entre o Profissional de Saúde e o Paciente, estabelecendo-se uma relação sólida de confiança recíproca (Albuquerque, 2013; Dias et al., 2015b; Monteiro, 2016).

A ansiedade e o medo vivenciados, que parecem constituir uma barreira comunicacional na prática clínica Odontopediátrica, podem ter origem em experiências Médico-Dentárias precedentes de natureza algo traumática, ou até nas próprias caraterísticas da personalidade da Criança, podendo configurar-se como o principal desencadeante de comportamentos não colaborantes (Dias et al., 2018a; Martins \& Dias, 2016; Mika et al., 2018; Reis et al., 2008; Silva, 2018).

O rosto Humano, mais concretamente a cavidade oral, não só é a parte do corpo humano que melhor espelha ações e expressões emocionais, como também se constitui como o foco do tratamento odontopediátrico. Deste modo, o Médico Dentista deve tornar-se um verdadeiro leitor do rosto dos pacientes, dado que trabalha essencialmente com doentes que se podem enquadrar na categoria de doentes emudecidos (Dias et al., 2018c),visto que, o ato terapêutico, ao ser executado na 
cavidade oral, impossibilita, naturalmente, os pacientes de se expressarem verbalmente (Albuquerque, 2013; Dias et al., 2018b; Dias et al., 2018c).

A trajetória terapêutica e as etapas que lhe são inerentes, assim como o setting terapêutico, são pontos de partida essenciais para a compreensão da relação Médico-Paciente e da triangulação terapêutica que se instala nas consultas de Odontopediatria, pois na maior parte das consultas, o Paciente de primeira ordem (criança) é acompanhado por um Paciente de segunda ordem, nomeadamente por um familiar próximo (Mota-Cardoso, 2012; Silva, 2018).

Pretende-se com o presente artigo abordar problemáticas inerentes aos atos pedagógicos em contextos clinico-terapêuticos, ou seja, o recurso a estratégias de Educação para a Saúde, bem como os conceitos de Empowerment e Enabling associados às estratégias de ensino ao Doente no decurso da trajetória terapêutica em Odontopediatria. No mesmo registo, serão apresentados instrumentos pedagógicos de Educação para a Saúde desenvolvidos, propositadamente, para o simulacro do presente estudo de caso.

\section{Procedimentos metodológicos no âmbito da educação para a saúde}

No âmbito da consulta de Odontopediatria, o recurso a instrumentos lúdico-pedagógicos permite ampliar o pensamento simbólico da criança, funcionando, também, como um suporte relacional por excelência na relação terapêutica, constituindo-se como um meio privilegiado para entrar em contato com a criança (Dias et al., 2015a; Mota-Cardoso, 2012).

No presente estudo de caso, foram utilizados vários instrumentos de Educação para a Saúde, elaborados com recurso a pictogramas, em formato de brochuras e/ou macromodelos, tendo como objetivo a promoção da higiene oral e a prevenção da carie dentária, consciencializando a criança acerca dos diferentes comportamentos salutogénicos a adotar no futuro para uma boa saúde oral. Estes instrumentos pedagógicos são adaptados especificamente para crianças na fase de latência, verificando-se, neste estádio de desenvolvimento, uma predisposição desenvolvimentista para a elaboração de desenhos como instrumentos de recurso ao imaginário infantil (Corrêa \& Pinheiro, 2013; Menezes et al., 2008; Sintra, 2018; Sousa \& Trindade, 2013; Steibel et al., 2011).

No mesmo registo, um pictograma é um símbolo gráfico universal utilizado para transmitir informações especificas, independentemente do idioma, da capacidade intelectual ou do conhecimento técnico do observador (Chernoff, 1973; Dranka, 2012; Magalhães et al., 2013). O símbolo designado como pictograma refere-se a um tipo particular de comunicação pictórica que, ao combinar figuras e conceitos permite transmitir, instantaneamente, informações marcantes no âmbito da prática social e da vivência comunitária. Uma vez que visualizar informações ao invés de lê-las, é mais célere, direto e fácil de recordar, os pictogramas valem-se da simplificação, acentuando a clarividênciados factos essenciais da mensagem visual e pictográfica (Barros et al., 2013; Clawson et al., 2012; Dranka, 2012; Hameen-Antillaet al., 2004; Medeiros et al., 2011).

O antropomorfismo, presente em alguns pictogramas, é definido como a atribuição de motivações, emoções e capacidades cognitivas consideradas tipicamente humanas a objetos não-humanos, tornando-se possível porque os seres humanos são capazes de percecionar características psicológicas a determinados objetos inanimados (Chernoff, 1973; Magalhães et al., 2013; Mattos, 2013). De acordo com esta premissa básica, os instrumentos elaborados foram suportados com base nos estudos desenvolvidos por Chernoff (1973) e Magalhães et al. (2013).

Nesta faixa etária, uma das problemáticas consideradas é a transição da dentição decídua para a definitiva. De acordo com este objetivo, foi elaborado um estudo de Dias et al. (2019b),em que as crianças foram incentivadas a desenhar a forma como percecionavam o seu rosto, antes e depois da perda dos dentes de leite e a responder a questões abertas de, como se sentiam, antes e depois desta ocorrência. De acordo com os resultados obtidos na primeira fase, as crianças representavam o seu rosto nos perceptos desenhados com um sorriso isento de diastemas ou lacunas, contrariamente, ao momento da perda dos dentes decíduos (segunda fase), em que as crianças 
desenharam, na sua maioria, a sua boca com ausência de peças dentárias, com a presença de diastemas, curiosamente referidos na análise de conteúdo das respostas escritas- como balizas (Dias et al., 2019b). Ainda de acordo com este estudo, uma das consequências da perda dos dentes decíduos pode ser inconscientemente percecionada pela criança como uma "pequena morte do Eu", conotada com a autoimagem e a sua qualidade de vida (Santos, 2017).

Neste sentido, foi desenvolvido um pictograma que representa a sequência cronológica da erupção da dentição definitiva (Ver figura $\mathrm{N}^{\circ} 1$ ). Esta banda desenhada é constituída por 5 secções: na i) primeira, encontram-se três dentes felizes, ao contrário do que sucede no ii) segundo pictograma, onde o dente do meio se encontra extruído face aos outros, denunciando uma expressão assustada. Já no iii) terceiro pictograma, o mesmo dente encontra-se ausente, simbolizando a queda do dente decíduo. No entanto, ao abrir a janela subjacente ao mesmo, é revelado o dente definitivo, como forma de explicar à Criança que, iv) a ausência desta peça dentária não é de caracter permanente e que, lentamente, irá emergir um novo dente, representado no quarto pictograma. Por fim, voltam a estar presentes três dentes, iv) sendo o dente definitivo representado como um dente maior, forte e robusto.

Figura 1. Sequência cronológica da erupção da dentição definitiva

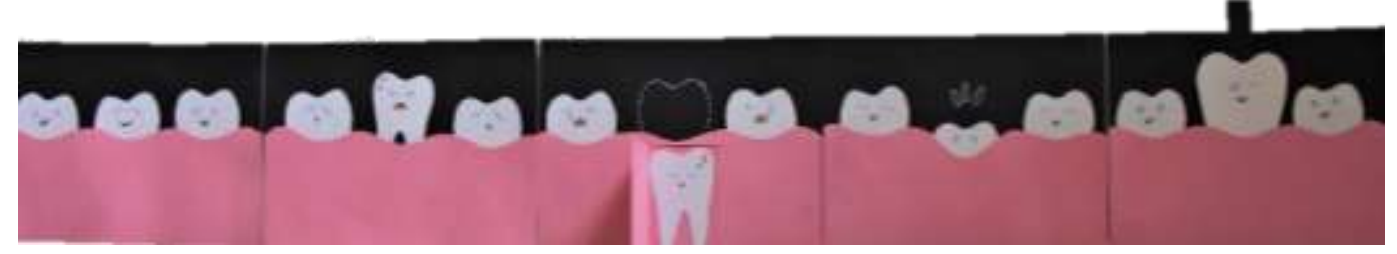

Fonte: Elaboração própria.

Com o intuito de consciencializar não só a Criança, mas também os Pacientes de $2^{\mathrm{a}}$ ordem, sobre a importância da técnica de escovagem na prevenção da cárie dentária, foi desenvolvido um macromodelo representativo da cavidade oral (Ver figura $\mathrm{N}^{\circ} 2$ ). No macromodelo, não só se encontram representados os dentes, por copos e taças de plástico, mas também a língua, reforçando a ideia de que nenhuma superfície da cavidade oral deve ficar isenta de uma boa higienização. É ainda acompanhado pelo fio dentário, enfatizando a importância da limpeza interproximal e, por uma escova de dentes, instrumento ao qual se recorreu para a exemplificação da técnica de escovagem adequada (Batalha, 2016). Para além da exemplificação da técnica, foi ainda dada a indicação da frequência, abrangência e tempo de escovagem. De referir ainda que, estes dois últimos instrumentos-acessórios, fio dentário e escova, apresentam um tamanho proporcional ao do macromodelo.

Figura 2. Macromodelo da cavidade oral

Fonte: Elaboração própria.

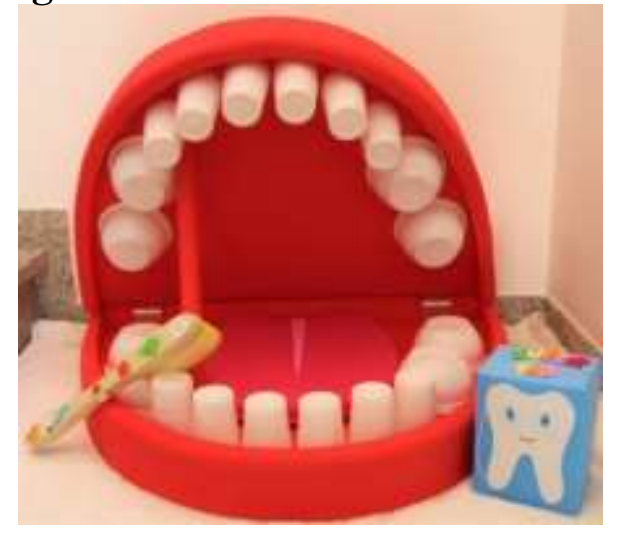


Ao trabalhar com uma Criança é muito importante criar um vinculo terapêutico de confiança básica, que se opõe à imagem estereotipada e tradicionalista criada do Médico Dentista como o arranca dentes (Dias et al., 2006). Torna-se, assim, fundamental (re)elaborar as imagens mentais instaladas e explicar as ferramentas clínicas, o material e a profissão do Médico Dentista, recorrendo à atividade lúdica e desenhos como representações simbólicas da realidade clínico-terapêutica (Dias \& Simões, 2016; Mota-Cardoso, 2012).

Nesta linha de registo, constitui-se como transversal a todo o ato pedagógico o recurso a um macromodelo sobre o setting terapêutico, o qual tem como objetivo sensibilizar a Criança para o setting terapêutico, desmistificando, assim, alguns mitos e medos instalados. Este macromodelo apresenta-se com cinco caixas incorporadas e acopladas a janelas com badanas, que vão sendo destapadas/abertas pela criança sequencialmente, revelando o seu conteúdo simbólico e pictórico (Ver figura No3).

Figura 3. Macromodelo sobre o setting terapêutico

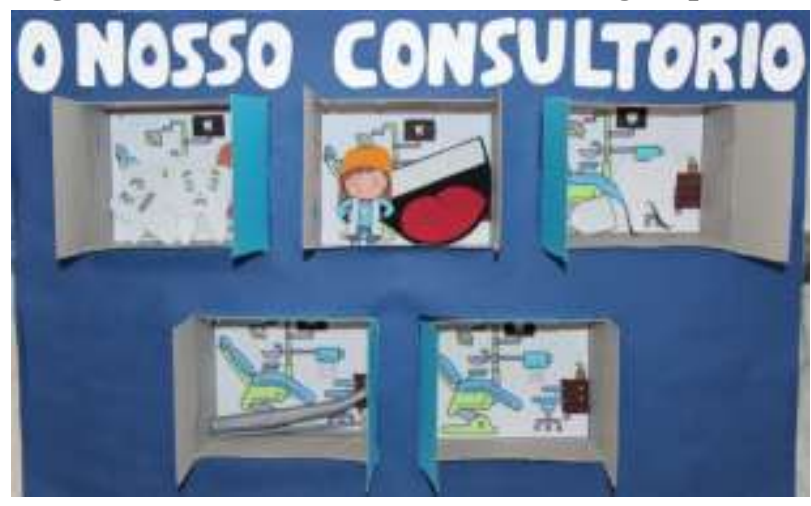

Fonte: Elaboração própria com base em Dias et al. (2018a).

Ao abrir a primeira janela (Ver figura $\mathrm{N}^{\circ} 4$ ), aparece a figura de uma menina e, cinco dentes antropomorfizados que pretendem representar as emoções vivenciadas no setting terapêutico (Ver figura $\mathrm{N}^{\circ}$ 5). No início da consulta, a Criança é questionada pelo Médico Dentista sobre com qual dos dentes antropomorfizados, mais se identifica emocionalmente, permitindo ao Médico Dentista compreender de forma simbólica as suas vivências emocionais (Mota-Cardoso, 2012).

Figura 4. A criança: Representação da primeira janela do macromodelo

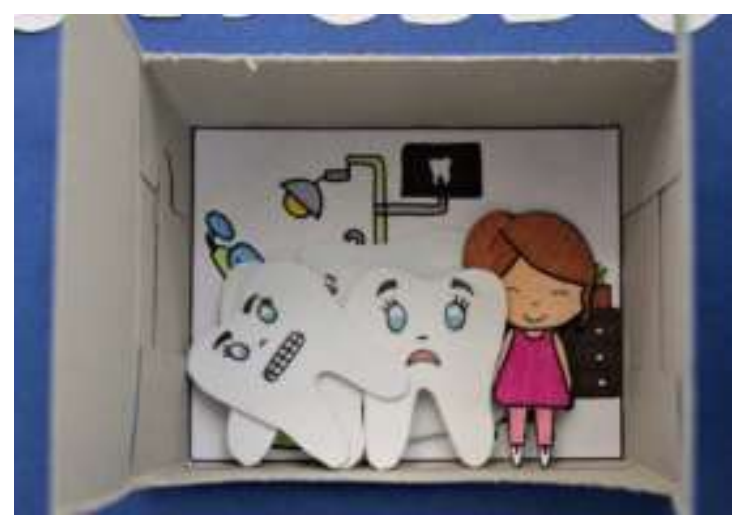

Fonte: Elaboração própria com base em Chernoff (1973). 
Figura 5. Dentes antropomorfizados pertencentes à primeira janela

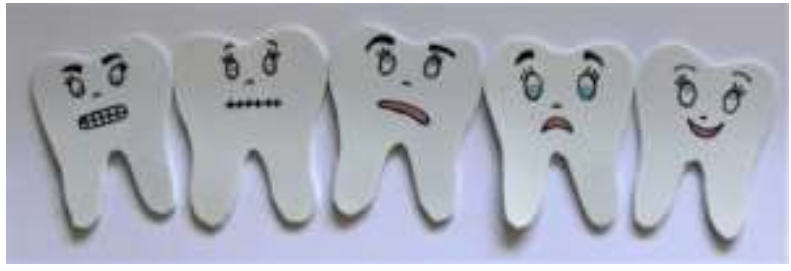

Fonte: Elaboração própria com base em Chernoff (1973).

No final da consulta, a Criança é novamente questionada sobre qual dos dentes antropomorfizados mais se identifica emocionalmente permitindo, assim, avaliar, subjetivamente, a trajetória das suas vivências no decurso da consulta.

No interior da segunda janela (Ver figura $\mathrm{N}^{\circ} 6$ ) encontramos uma figura alusiva ao Médico Dentista e outra alusiva à cavidade oral, sendo o rosto do Médico Dentista desenhado com um sorriso aberto, procurando, de forma simplista, associar este Profissional de Saúde a emoções positivas.

Figura 6. O Médico Dentista: Representação da segunda janela do macromodelo

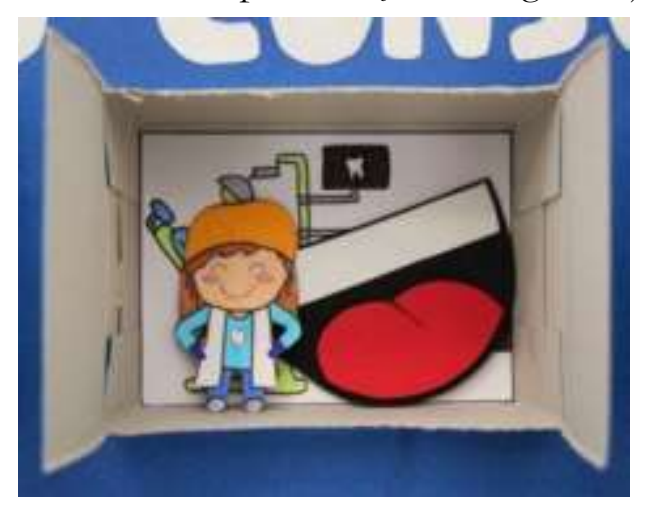

Fonte: Elaboração própria com base em Dias et al. (2006).

As janelas seguintes são direcionadas para o ato terapêutico em si, dando a conhecer alguns dos instrumentos e espaços com que a Criança se irá deparar ao longo da trajetória terapêutica. Neste sentido, na terceira janela (Ver figura $\mathrm{N}^{\circ}$ ) está presente a figura de uma ampola radiográfica e de um dente hígido que, ao levantar as suas raízes, revela o seu interior, permitindo explicar à Criança o objetivo do Raio-X (Ver figura $\mathrm{N}^{\circ}$ ).

Figura 7. Sala de Raio-X: Representação da terceira janela do macromodelo

Fonte: Elaboração própria.

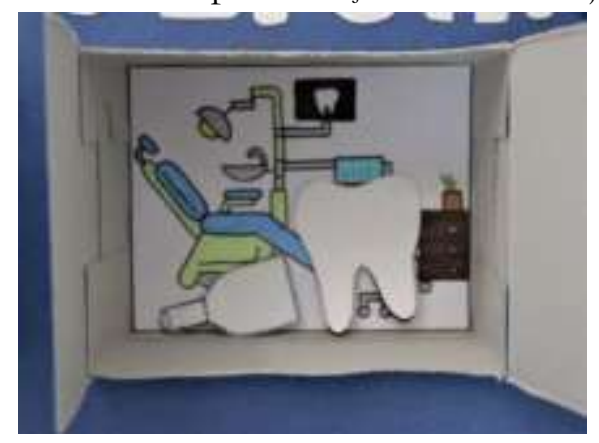


Figura 8. Ampola radiográfica e representação de um dente radiografado

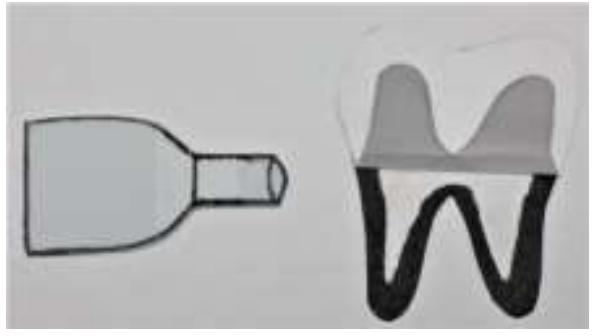

Fonte: Elaboração própria.

A quarta janela (Ver figura $\mathrm{N}^{\circ}$ ) ) representa o espaço considerado como o mais assustador para a Criança- o gabinete do Médico Dentista. Recorremos a madeira e a outros materiais recicláveis para recriar alguns instrumentos utilizados no ato clínico, tais como: a sonda, o espelho e a turbina. Com recurso a eufemismos (v.g. comparação do som da turbina ao som de um aspirador), os instrumentos, e as suas respetivas funções, vão sendo dados a conhecer à criança.

Figura 9. Instrumentos Clínicos: Representação da quarta janela do macromodelo

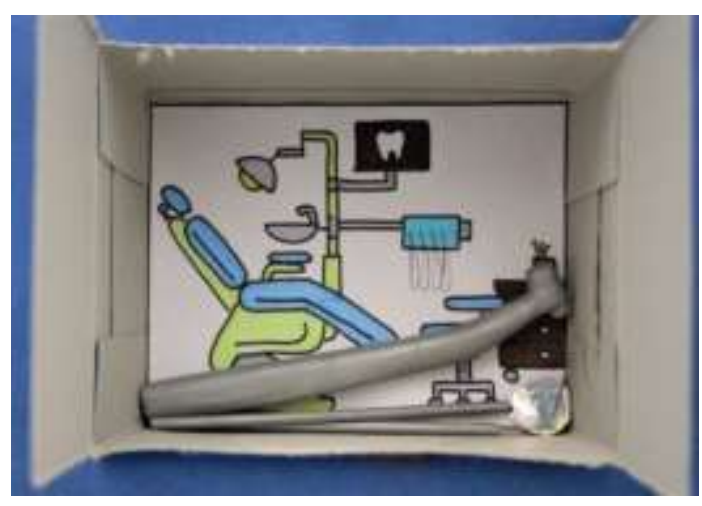

Fonte: Elaboração própria.

O objetivo destes instrumentos de Educação para a Saúde tem como foco estratégico a adesão terapêutica do Paciente e a desmistificação do medo antecipatório, recorrendo ao conceito empírico de Empowerment. O termo Empowerment é designado pela Organização Mundial de Saúde (OMS, 2001) como um processo contínuo, no qual indivíduos e/ ou comunidades adquirem e ganham confiança, autoestima, compreensão e poder necessários para articular os seus interesses, e, mais ampliadamente, ganharem um certo controlo cognitivo e emocional das suas vidas (Ramos, 2001).

Relativamente aos cuidados de saúde, o Empowerment é contextualizado como uma interação entre três elementos: o indivíduo, enquanto elemento de uma sociedade, os prestadores de cuidados de saúde, e as políticas de saúde / educativas. Esta triangulação, aparentemente desconexa, poderá facilitar ou não a implementação de princípios básicos advogados pelo nomeado mecanismo de Empowerment (Menon, 2002), os quais são fundamentais para capacitar as crianças para a adoção de comportamentos salutogénicos, conceito este designado por Enabling (Botas, 2015; Dias et al., 2015b).

No presente estudo de caso, o Empowerment e Enabling são evidenciados no decurso do ato pedagógico, sendo apresentados instrumentos de EPS em forma de pictogramas e macromodelos como suporte do ato de ensino à díade Pais-Criança, que, ao longo da trajetória terapêutica, vão progressivamente adotando um perfil de Doente(s)informado(s). 


\section{Conclusões}

O desenvolvimento de capacidades comunicativas e relacionais é substancial ao nível da formação pré e pós-graduada em Medicina Dentária. Neste registo, as aquisições de ferramentas comunicacionais ministradas ao nível dos conteúdos programáticos das unidades curriculares das formações em Medicina Dentária constituem-se como notoriamente relevantes, não só ao nível do contacto terapêutico e relacional com os pacientes, bem como com o trabalho em equipa com os colegas e outros Profissionais de Saúde.

Por outro lado, a criação de instrumentos lúdico-pedagógicos privilegia a relação Médico DentistaCriança, permitindo reduzir o "peso psicológico" da vivência do medo e da ansiedade. De um modo geral, o recurso a pictogramas e macromodelos em contextos de saúde, permitem explicar, de forma lúdica e simplificada, os motivos da consulta e o papel do Médico Dentista, incentivando, também, a alteração de hábitos e comportamentos outrora considerados desadequados, podendo, assim, serem eliminadas algumas barreiras que se instalam ao nível da comunicação relacional e pedagógica desta díade relacional.

Na prática clínica, os pictogramas e os macromodelos são utilizados para um melhor entendimento e memorização das informações prestadas nos atos pedagógicos (atos de ensino), no entanto, estas informações devem ser sempre acompanhadas de uma panóplia de outros atos informativos, tendo em consideração o nível de literacia do Paciente e dos seus acompanhantes.

Da mesma forma que os mecanismos Empoderamento e Capacitação permitem a capacitação e o empoderamento das capacidades dos Pacientes recorrendo a instrumentos de Educação para a Saúde, o role playing, enquanto estratégia de ensino-aprendizagem dos Discentes permite, também, a aquisição de capacidades comunicacionais dos Discentes enquanto futuros Médicos Dentistas. Parece ser consentâneo que o roleplaying enquanto estratégia de ensino-aprendizagem permite que os Discentes construam conhecimento sobre as suas (auto) capacidades comunicacionais a partir de uma reflexão crítica das suas próprias experiências e/ou dos seus pares. No mesmo registo, esta estratégia de ensino aprendizagem, permite que a reflexão cognitiva emocional da Díade se interiorize, tanto a partir da perceção do perfil do Médico Dentista como da perceção do perfil do Paciente, contribuindo assim, para a humanização dos cuidados de saúde prestados.

O roleplaying permite, ainda, uma vivência intrapsíquica mais adequada da transição vivencial do discente de um cenário fictício, em sala de aula, para o um cenário real, que irá ser vivenciado no futuro no consultório dentário, destacando-se, assim, a importância deste tipo de simulacros pedagógicos antes da ocorrência de um primeiro contacto direto com um paciente. Podemos então concluir que, de acordo com o presente simulacro do estudo de caso elaborado, que mais importante que o simulacro em si, é a observação sistemática dos Discentes, análise, discussão e compreensão dos dados obtidos para uma futura aquisição e sedimentação dos conhecimentos adquiridos.

\section{Referências bibliográficas}

Albuquerque, Mafalda Martins (2013). Estratégias de comunicação na relação médico doente em medicina dentária. Tese de Mestrado em Medicina Dentária. Universidade de Lisboa. Portugal. Retirado

de https://repositorio.ul.pt/bitstream/10451/25444/1/ulfmd03046 tm Mafalda Albuquer que.pdf Consulta: 09/06/2020.

American Academy of Pediatric Dentistry (2014). Guideline on infant oral health care. Pediatric Dentistry. Volume 36, No. 6. EUA (Pp. 1141-1145).

American Dental Association (2000). Statement on early childhood caries. Retirado de https://www.ada.org/en/about-the-ada/ada-positions-policies-andstatements/statement-on-early-childhood-caries. Consulta: 09/06/2020. 
Araújo, Monica Martins Trovo; Silva, Maria Júlia Paes; Puggina, Ana Cláudia (2007). A comunicação não-verbal enquanto fator iatrogénico. Revista da Escola de Enfermagem da USP. Volume 41, No. 3. Brasil (Pp. 419-425).

Armelin, Cláudia Batagin; Wallau, Rodrigo Ambros; Sarti, Cynthia Andersen; Pereira, Sonia Regina (2005). A comunicação entre os profissionais de pediatria e a criança hospitalizada. Revista Brasileira de Crescimento e Desenvolvimento Humano. Volume 15, No. 2. Brasil (Pp. 45-54).

Barros, Izadora; Alcântara, Thaciana; Mesquita, Alessandra; Santos, Anne; Paixão, Felipe; Lyra, Divaldo (2013). The Use of Pictograms in the Health Care: A Literature Review. Social and Administrative Pharmacy. Volume 10, No. 5. Brasil (Pp. 1-16).

Batalha, Joana Galego da Vila Mona (2016). A eficácia da escovagem em pacientes especiais através das escovas modificadas. Tese de Mestrado em Medicina Dentária. Universidade Católica Portuguesa. Portugal. Retirado de https://repositorio.ucp.pt/bitstream/10400.14/22212/1/Monografia\%20Joana\%20Batal ha\%202016.pdf Consulta: 09/06/2020.

Botas, Philippe (2015). Capacitação: Uma Ciência com Arte. Revista ADSO. Volume 1, No. 1. Portugal (Pp. 13-16).

Chernoff, Herman (1973). The use of faces to represent points in k-dimensional space graphically. Journal of the American Statistical Association. Volume 68, No. 342. EUA (Pp. 361 368).

Clawson, Terri; Leafman, Joan; Nehrenz, Guy; Kimmer, Sandra (2012). Using Pictograms for Communication. Military Medicine. Volume 177, No. 3. Inglaterra (Pp. 291-295).

Comassetto, Marcela Obst; Baumgarten, Alexandre; Kindlein, Katherine de Andrade; Hilgert, Juliana Balbinot; Figueiredo, Márcia Cançado; Faustino-Silva, Daniel Demétrio (2019). Acesso à saúde bucal na primeira infância no município de Porto Alegre, Brasil. Ciência e Saúde Coletiva. Volume 24, No. 3. Brasil (Pp. 953-961)

Corrêa, Crístia Rosineiri Gonçalves Lopes; Pinheiro, Glaúcia da Silva (2013). Período de Latência e tempo para compreender nas aprendizagens. Psicologia em Estudo. Volume 18, No. 1. Brasil (Pp. 61-69)

Dias, Maria do Rosário; Amorim, Armanda; Freches, Alexandra.; Guilherme, João (2006). Masked dentist in therapeutic setting. EACH 2006 - International Conference in Healthcare Abstracts. Almada, Portugal.

Dias, Maria do Rosário; Cruz, Catarina Vilarinho; Carvalho, Ana Reis (2015a). "Barnabé e sua aventura": Um projeto de educação para a saúde em disfonia infantil. Distúrbios Comum. Volume 27, No. 2. Brasil (Pp. 293-298).

Dias, Maria do Rosário; Cruz, João Amaral; Martins, Nádia Leitão (2015b). I am favolas: A health education instrument in dentistry. Journal Of Human Growth and Development. Volume 25, No. 3. Brasil (Pp. 325-330).

Dias, Maria do Rosário; Monteiro, Ana Lúcia; Naben, Letícia; Sobral, Adriana; Neves, Ana Cristina (2018a). No consultório do Odontopediatra: Um manual de Educação para a Saúde Oral. OMNIA. Volume 8, No. 3. Portugal (Pp. 35-41)

Dias, Maria do Rosário; Naben, Letícia Garcia; Monteiro, Ana Lúcia; Ferreira, Ana; Alves, Valter Pedroso; Delgado, Ana (2018b). When the Silence Speaks: The Smile. Journal of Biosciences and Medicines. Volume 6, No. 11. (Pp. 13-20).

Dias, Maria do Rosário; Naben, Letícia; Ferreira, Ana; Mendes, José João (2018c). The Language of Silence In The Therapeutic Setting Of Dental Medicine. Advances in social Sciences Research Journal. Volume 5, No. 12. Inglaterra (Pp. 351-362).

Dias, Maria do Rosário; Naben, Letícia; Ventura, Irene; Coroado, Sofia; Dias, Viriato; Santos, Sofia (2019a). The house of the little tooth Diniz: an oral health educational project. Children and Teenagers. Volume 2, No. 1. EUA (Pp. 60-71). 
Dias, Maria do Rosário; Santos, Ana Catarina; Naben, Letícia; Ventura, Irene (2019b). Dental Decay in the Change of Deciduous Teeth: The Child's Self-Perception. EC Dental Science. Volume 18, No. 9. Inglaterra (Pp. 2214-2220).

Dias, Maria do Rosário; Sebastião, Fátima Joana de Campos; Silva, Maria Matilde Rosa Tomaz Pinto (2014). Comunicação pedagógica e relacional em uma consulta periodontológica. Revista Periodontia. Volume 24, No. 4. Brasil (Pp. 50-56).

Dias, Maria do Rosário; Simões, Nádia (2016). On the Mental Representation of (Un)Healthy Tooth: (Un)Healthy Tooth Profiles among Children. Journal of Educational and Developmental Psychology. Volume 6, No. 1. Canadá (Pp. 110-116).

Dougall, Allison; Fiske, J. (2008). Access to special care dentistry, part 2. Communication. British Dental Journal. Volume 205, No.1. Inglaterra. (pp. 11-21).

Dranka, Lucas Kitt. (2012). Pictogramas: Teoria, Desenvolvimento e Aplicação. Tese de Mestrado. Universidade Federal do Paraná. Brasil. Retirado de http://www.ciclovida.ufpr.br/wp-content/uploads/2013/03/Relatório-Lucas-K.Dranka-Pictogramas-Teoria-Desenvolvimento-e-Aplicação.pdf Consulta: 09/06/2020.

Graça, Adriana Vivas (2012). A importância da primeira "ida ao dentista": um estudo exploratório a partir dos desenhos das crianças. Tese de Mestrado de Medicina Dentária. Universidade do Porto. Portugal. Retirado de https://repositorioaberto.up.pt/bitstream/10216/87648/2/161062.pdfConsulta: 09/06/2020.

Hameen-Antilla, Katri; Kemppainen, Kati; Enlud, Hannes; Patricia, J Bush; Marja, Airaksinen (2004). Do pictograms improve children's understanding of medicien leaflet information?.Patient Education and Couseling.Volume 55, Noruega (Pp. 371-378).

Lemos, Letícia Vargas Freire Martins; Myaki, Silvio Issáo; Walter, Luiz Reynaldo de Figueiredo; Zuanon, Angela Cristina Cilense (2014). Promoção da saúde oral na primeira infância: idade de ingresso em programas preventivos e Aspectos comportamentais. Einstein. Volume 12, No. 1. Brasil (Pp. 6-10).

Magalhães, Luanna Marques; Nina-e- Silva, Cláudio Herbert; Alvarenga, Lenny Francis Campos (2013). Atribuição de emoções a traços faciais artificiais. Revista Da Universidade Vale Do Rio Verde. Volume 11, No. 2. Brasil (Pp. 462-469)

Martins, Nádia; Dias, Maria do Rosário (2016). Contágio emocional da ansiedade encarregado de educação/criança em odontopediatria. Revista Portuguesa de Estomatologia, Medicina Dentária e Cirúrgia Maxilofacial. Volume 57, No. 3. Portugal. (Pp. 164-170).

Mattos, Alexandre Almeida Juruena (2013). Antropomorfismo na Cultura da Animação. Programa de Pós-Graduação em Estudos Contemporâneos. Universidade Federal Fluminense. Brasil. Retirado de http://www.artes.uff.br/dissertacoes/2013/alexandrejuruena.pdf Consulta: 09/06/2020.

Medeiros, Giovanna Christinne Rocha; Silva, Priscila Queiroz; Silva, André Santos; Leal; Leila Bastos (2011). Pictogramas na orientação farmacêutica: Um estudo de revisão. Revista Brasileira de Farmácia. Volume 92, No.3. Brasil (Pp. 96-103)

Menezes, Marina; Moré, Carmen; Cruz, Roberto Moraes (2008). O desenho como instrumento de medida de processos psicológicos em crianças hospitalizadas. AvaliaçãoPsicológica. Volume 7, No. 2. Brasil (Pp. 189-198).

Menon, Sanjay (2002). Toward a model of psychological health empowerment: implications for health care in multicultural communities. Nurse Education Today. Volume 22, No. 1. (Pp. 28-39)

Mika, A.; Mitus-Kenig, M.; Zeglen, A.; Drapella-Gasior, D.; Rutkowska, K.; Josko-Ochojska, J. (2018). The child's first dental visit. Age, reasons, oral health status and dental treatment needs among children in Southern Poland. European Journal of Paediatric Dentistry. Volume 19. No. 4. Itália (Pp. 265-270). 
Monteiro, Ana Sofia Feijão (2016). Gestos e sinais na consulta de medicina dentária. Tese de Mestrado em Medicina Dentária. Instituto Superior de Ciências da Saúde Egas Moniz. Portugal. Retirado de https://comum.rcaap.pt/bitstream/10400.26/17435/1/Monteiro Ana Sofia Feij\%c3\% a3o.pdf Consulta: 09/06/2020.

Mota-Cardoso, Rui (Ed.) (2012). Competências Clínicas de Comunicação. Universidade do Porto. Portugal.

Organização Mundial de Saúde. (2001). Community participation in local health and sustainable development. European Sustainable development and Health Series, 4.

Ramos, Ana Lúcia (2001). Empoderamento em Saúde: o papel do Enfermeiro. The internet Journal of Healthcare Administration. Volume 1, No. 2. EUA (Pp. 26-31).

Ramos, Ana Paula; Bortagarai, Francine Manara (2012). A comunicação não-verbal na área da saúde. Revista CEFAC. Volume 14, No. 1. Brasil (Pp. 164-170).

Reis, Fátima; Dias, Maria do Rosário; Leal, Isabel (2008). A consulta no setting odontopediátrico: A percepção subjetiva do medo. Análise Psicológica. Volume 2, No. 26. Portugal (Pp. 239-250).

Santos, Susana Biscaia (2017). A Auto-perceção Da Criança Sobre a Perda Da Dentição Decídua Em Odontopediatria. Tese de Mestrado em Medicina Dentária. Instituto Superior de Ciências da Saúde Egas Moniz. Portugal. Retirado de https://comum.rcaap.pt/bitstream/10400.26/20002/1/Santos Susana Biscaia dos.pdf Consulta: 09/06/2020.

Silva, Marta Gomes Mendes Alves (2018). Presença ou Ausência dos pais na consulta odontopediátrica. Tese de Mestrado em Medicina Dentária. Universidade Fernando Pessoa. Portugal. Retirado de https://bdigital.ufp.pt/bitstream/10284/7306/1/PPG 29385.pdf Consulta: 09/06/20.

Sintra, Ana Catarina Pinto (2018). A participação ativa da criança no processo de ensinoaprendizagem. Tese de Mestrado em Educação Pré-Escolar. Instituto Piaget. Portugal. Retirado

de https://comum.rcaap.pt/bitstream/10400.26/23886/1/Ana\%20Catarina $\% 20 \operatorname{Sintra} \% 20$ \%20ESE.pdf Consulta: 09/06/2020.

Sousa, Rita Ramos; Trindade, Rui (2013). O impacto da saúde escolar na comunidade educativa. Educação, Sociedade \& Cultura. Volume 38. Portugal (Pp. 99-116).

Steibel, Denise; Hallberg, Ana Elisa; Sanchotene, Bianca; Campezatto, Paula von Mengden; Silva, Milena da Rosa; Nunes, Maria Lúcia Tiellet (2011). A latência na atualidade: considerações sobre crianças encaminhadas para psicoterapia. Aletheia. Volume. 35, No. 36. Brasil (Pp. 51-68). 